\title{
Commentary
}

\section{Systematic infection control in Greek physiotherapy practices during the COVID-19 pandemic}

\author{
Eleftherios Paraskevopoulos* and Maria Papandreou \\ Department of Physiotherapy, University of West Attica, Athens, Greece
}

Received 10 May 2020

Accepted 16 May 2020

\section{Introduction}

The COVID-19 pandemic has affected largely the entire health care system and especially physiotherapy services. It is known that in most cases, physiotherapy treatments require an interaction between therapist and the patient in close proximity, especially in manual therapy approaches. It should be stated that since March, the Panhellenic Association of Physiotherapists in Greece has provided specific guidelines to all registered physiotherapists for the protection of clients and physiotherapists from COVID-19. In these guidelines, physiotherapists were advised to provide face to face physiotherapy services only to urgent cases based on their clinical judgement. However, the rapid spread of the infection worldwide left the physiotherapists, in many cases, with the unanswered question as to whether some treatment sessions could be postponed or even cancelled, based on the health care needs of the client and without considering the financial burden that these cancellations may cause for private physiotherapy practices. Undoubtedly, health of all

\footnotetext{
*Address for correspondence: Eleftherios Paraskevopoulos, University of West Attica, Athens, Greece, Ag. Spiridonos 28, 12243, Athens, Greece. E-mail: lefteris.15@hotmail.com.
}

patients should be prioritized, however, governmental long-term financial support measures should be implemented to ensure practice viability.

The architectural setting of all private physiotherapy outpatient clinics in Greece is mainly controlled by the Ministry of Health based on the national guidelines that were published in the Official Government Gazette ( $\Phi$ EK 1009/B/2013). According to the Greek Law, every legal private physiotherapy practice should have a total space of at least $70 \mathrm{~m} 2$ which includes: a patient waiting room, toilet with sink and patient treatment areas. Patient treatment areas should be properly designed to include separate areas of electrotherapy, mechanotherapy, kinesiotherapy, therapeutic exercise and hydrotherapy. However, these designated treatment areas are not adequate in order to protect clients and therapists from the possibility of infection from COVID-19. Moreover, a substantial number of physiotherapy practices separate treatment tables with curtains. This creates an ideal environment for contamination of other patients and should not be allowed at this stage.

It is now widely accepted that the only way to prevent the increase of COVID-19 infection rates is social distancing. In fact, Greece was one of the countries that managed to properly "flatten the curve" substantially well, in comparison with other countries 
and maintained a low number of new cases until today. This was the result of extreme but necessary social distancing measures such as closures of workplaces, schools and universities, cancellations of mass gatherings and the quarantine of areas that were affected into a large extend by the virus. Furthermore, on the $23^{\text {rd }}$ of March the Prime Minister of Greece announced the full lockdown, to stop the spread of the virus until the $4^{\text {th }}$ of May.

The aforementioned measures have affected physiotherapists as professionals and the services that they were able to provide. Although regulations were put into effect that allowed physiotherapy practices to provide treatment to "urgent" cases such as in post-surgical patients, physiotherapists are still facing difficulties deciding which patients are really the "urgent cases" based not only on their musculoskeletal post-surgical stage but also on a risk to benefit ratio, as the possibility of infection is increased in closed settings [1]. Recent evidence has shown that all people aged 65 years and over are at high risk for severe illness [2]. Since older people are at higher risk of undergoing surgery for musculoskeletal conditions [3], there is now the possibility of delayed rehabilitation in this population and the development of inequalities in access to proper healthcare [4].

Apart from the post-surgical cases, a large number of physiotherapists provide treatment to several other people with long-term health care problems. Physiotherapy to these patients has now been postponed without knowing when it will be safe for physiotherapists to restart their usual treatment care, as these are patients with underlying medical conditions with a higher risk of developing severe illness from COVID19. This can be even more complicated when we consider that some of these patients receive treatment in nursing homes and long-term care facilities. These settings have been recognized as high-risk, for severe outcomes from COVID-19 outbreaks [5].

Not only patients, but also physiotherapists are at an increased risk of being infected from COVID19. Thus, all physiotherapists should follow specific guidelines to protect patients and themselves from infection. At this point it should be stated that symptomatic and asymptomatic patients from COVID-19 have shown resemble viral load [6]. Furthermore, recent evidence has shown that viral shedding of patients with COVID-19 peaked on or before symptom onset and a substantial proportion of transmission may occur before symptom onset [7]. This is especially important in the case of younger asymptomatic physiotherapists who treat patients in the outpatient setting as their transmission potential is still highly possible and the guidelines should be followed irrespective of their symptomatology [6].

It is important at this time to understand, as physiotherapists, that the pandemic should not alter treatment protocols or skip treatment approaches in order to avoid close proximity with patients. Furthermore, inappropriate use of accelerated exercise protocols or excessive use of electrotherapy modalities to avoid contact, that expose patients to further injury and lack evidence should not be acceptable at any stage of the pandemic. However, proper protective equipment should be used by physiotherapists including gloves, gowns, medical mask, and eye protection (goggles or face shields) during treatment [8].

\section{Disinfection control in physiotherapy practices}

According to recent guidelines by the World Health Organization [9] we propose several infection control measures for physiotherapy practices during the pandemic. With regards to the physiotherapy practice environment it is suggested that waiting rooms should be equipped with disinfectant suspenders. Use of the disinfectant should be suggested and hand disinfection procedures should be explained to patients, prior to arrival for their treatment session and preferably during appointment scheduling. Before scheduling any appointment, physiotherapists should advise patients to report any COVID-19 related symptoms such Cough, Shortness of breath, Fever, Chills, Repeated shaking with chills, Muscle pain, Headache, Sore throat, New loss of taste or smell [10]. Also, patients should be asked whether someone from their family had fallen ill or they had contact with other people that reported the aforementioned symptoms. In these cases, treatment should be postponed.

Patients arriving for physiotherapy should be aware, a priori, that they should remain outside the practice, in case there is another patient in the designated waiting room. Physiotherapists should also ensure that there is adequate time between the appointments to allow them to remove carefully all protective equipment and dispose it in a separate closed bin, wash their hands properly and disinfect all surfaces in the treatment room that were in contact with the patient. This is especially important during a pandemic, since prior research has identified several pathogenic microorganisms in the treatment tables of 
rehabilitation practices, including gram positive and gram negative organisms [11]. Furthermore, physiotherapists should provide enough time between sessions to open the windows inside the treatment room, in order to ventilate properly the area. Patients that arrive late for treatment, should know a priori that their appointment may be cancelled or a reduced treatment time may be provided to avoid crowding.

Physiotherapists should advise patients to wear a face mask when entering the practice without accompanying persons and provide a mask in case some patients do not own it. In case of severely disabled patients that need to be assisted by a caregiver or in the case of children, the accompanying person should also wear protective mask. All door handles should be touched only by the physiotherapist and disinfect these at a regular basis. The therapist should also inform patients to maintain a distance of at least one meter from the therapist. The therapist should be able to decide when it is necessary to provide manual treatments and the patient should be aware that direct contact will be reduced to a minimum.

In order to minimize the duration of stay inside the practice, physiotherapists should perform most part of the subjective examination through telephone or via a video call. A telerehabilitation setting may be of significant importance as part of the examination procedure, during the pandemic, for surgical patients [12]. Telerehabilitation may be also used to supervise patients on their exercises at home. It is also advised for physiotherapists to avoid providing in paper form, any exercise programmes to avoid re-contamination and use specially designed online software. Lastly, patients should disinfect their hands before leaving the practice, and remove their masks only outside the practice. Removal of the mask should be performed properly by handling at the ties and discard it in a closed bin. Also, due to possible contamination of cash, electronic payment should be provided at all times.

\section{Contaminated surfaces in physiotherapy practices}

Infection from COVID-19 via contaminated surfaces is a possible route for transmission [13]. Recently, the survival of COVID-19 in different surfaces has been examined [13]. The results showed that the virus may survive for up to 4 hours in copper and for up to 3 days on plastic and stainless steel [12]. Thus, establishing proper disinfection proce- dures inside physiotherapy practices is necessary, in order to reduce the risk of infection via fomites.

All disinfectants which are classified as Biocidal Products based on EU Regulations (No 528/2012) have not been studied for their effectiveness against the COVID-19. However, recent guidelines from the European Centre for Disease Prevention and Control [14] suggest that all virucidal disinfectants can be used for disinfection of surfaces in healthcare settings. Another option is $0.05 \%$ sodium hypochlorite (NaClO) $(1: 100$ dilution Chlorine) by using $10 \mathrm{ml}$ bleach to $990 \mathrm{ml}$ water. For surfaces that are sensitive to damage from sodium hypochlorite, ethanol-based products may be used with $70 \%$ concentration. Accelerated Hydrogen Peroxide $0.5 \%$ may also be used for physiotherapy equipment. In case of contaminated surfaces with body fluids and waste, such as vomit, diarrhea, mucus or feces, $20 \mathrm{ml}$ bleach to $980 \mathrm{ml}$ of water ( $1: 50$ dilution) should be used after cleaning the areas with soap and water [15]. Disinfection of surfaces, such as floors and walls, inside the physiotherapy practice, may be performed with Quaternary Ammonium Compounds. At last, all textiles should be washed with laundry detergent at $90^{\circ} \mathrm{C}$ and for sensitive textiles, bleach should be added [14].

Physiotherapy remains necessary for a good proportion of patients even during a pandemic. Proper measures should be adopted by physiotherapists in order to protect the community and patients, and continue providing their services to those in need. Some adaptations to treatment may be necessary in some cases, however rehabilitation protocols should be followed in order to ensure quality of our services and avoid the risk of permanent functional deficits in post-surgical patients [4].

\section{Conflict of interest}

The authors declare no conflicts of interest.

\section{Funding}

None to report.

\section{References}

[1] European Centre for Disease Prevention and Control. Risk assessment on COVID-19. [homepage on the Internet]. ECDC: Stockholm; 2020. [cited 2020 May 04]. Available from: https://www.ecdc.europa.eu/en/currentrisk-assessment-novel-coronavirus-situation. 
[2] Du R-H, Liang L-R, Yang C-Q, et al. Predictors of mortality for patients with COVID-19 pneumonia caused by SARSCoV-2: a prospective cohort study. Eur. Respir. J. 2020.

[3] Jarvholm B, Lewold S, Malchau H, Vingard E. Age, bodyweight, smoking habits and the risk of severe osteoarthritis in the hip and knee in men. Eur. J. Epidemiol. 2005;20(6):537-542.

[4] Boldrini P, Bernetti A, Fiore P. Impact of COVID-19 outbreak on rehabilitation services and Physical and Rehabilitation Medicine (PRM) physicians' activities in Italy. An official document of the Italian PRM Society (SIMFER). Eur. J. Phys. Rehabil. Med. 2020.

[5] McMichael TM, Currie DW, Clark S, et al. Epidemiology of covid-19 in a long-term care facility in King County, Washington. N. Engl. J. Med. 2020.

[6] Ling Z, Xu X, Gan Q, et al. Asymptomatic SARS-CoV-2 infected patients with persistent negative CT findings. Eur. J. Radiol. 2020;126.

[7] He X, Lau EHY, Wu P, et al. Temporal dynamics in viral shedding and transmissibility of COVID-19. Nat. Med. 2020.

[8] World Health Organization. Rational use of personal protective equipment for coronavirus disease (COVID-19) and considerations during severe shortages: interim guidance, 6 April 2020. World Health Organization;2020.

[9] World Health Organization. Infection prevention and control during health care when COVID-19 is suspected. [homepage on the Internet]. 2020. [cited 2020 May 04]. Available from: https://www.who.int/publicationsdetail/ infection-prevention-and-control-during-health-care-whennovelcoronavirus-(ncov)-infection-is-suspected-20200125.
[10] Lambert C, Stoicu S, Hendrix I, et al. 2020-04-25/26 DAILY UNM GLOBAL HEALTH COVID-19 BRIEFING. 2020.

[11] Evans MW, Breshears J, Campbell A, Husbands C, Rupert R. Assessment and risk reduction of infectious pathogens on chiropractic treatment tables. Chiropractic \& Osteopathy. 2007;15(1):8

[12] van Egmond MA, van der Schaaf M, Vredeveld T, et al. Effectiveness of physiotherapy with telerehabilitation in surgical patients: a systematic review and meta-analysis. Physiotherapy. 2018;104(3):277-298.

[13] van Doremalen N, Bushmaker T, Morris DH, et al. Aerosol and surface stability of SARS-CoV-2 as compared with SARS-CoV-1. N. Engl. J. Med. 2020;382(16):1564-1567.

[14] European Centre for Disease Prevention and Control. Disinfection of environments in healthcare and nonhealthcare settings potentially contaminated with SARS-CoV-2. [homepage on the Internet]. ECDC: Stockholm; 2020. [cited 2020 May 04]. Available from: https://www.ecdc. europa.eu/en/publications-data/disinfection-environmentscovid-19 Accessed May 04, 2020.

[15] British Columbia Centre for Disease Control Ministry of Health. Environmental Cleaning and Disinfectants for Clinic Settings. [homepage on the Internet]. 2020. [cited 2020 May 04]. Available from: http://www.bccdc.ca/healthprofessionals/clinical-resources/covid-19-care/infection-co ntrol 